\title{
Identification and Characterization of CircRNAs of Two Pig Breeds as a New Biomarker in Metabolism-Related Diseases
}

\author{
Ai Li Wanlong Huang Xiuxiu Zhang Lingli Xie Xiangyang Miao
}

State Key Laboratory of Animal Nutrition, Institute of Animal Sciences, Chinese Academy of Agricultural Sciences, Beijing, China

\author{
Key Words \\ CircRNA • Pig • Subcutaneous fat • Fat deposition • Lipid metabolism • Diseases
}

\begin{abstract}
Background/Aims: CircRNAs, as miRNA sponges, participate in many important biological processes. However, it remains unclear whether circRNAs can regulate lipid metabolism. This paper aims to study the molecular mechanism of fat deposition and provide useful information for the prevention and therapy of lipid metabolism-related diseases. Methods: CircRNA sequencing was performed to investigate the expression of circRNAs in the subcutaneous adipose tissues of Large White pig and Laiwu pig. The expression of circRNAs was further validated by quantitative reverse transcription polymerase chain reaction (qRT-PCR). Furthermore, circRNA-microRNAs (miRNA)-mRNA interaction networks were constructed using bioinformatics tools. In addition, GO and KEGG enrichment analyses were performed for the target genes of circRNAs. Results: In the subcutaneous adipose tissue of Laiwu pig, 70 up-regulated circRNAs and 205 down-regulated circRNAs were identified. Two circRNAs (up-regulated circRNA_26852 and down-regulated circRNA_11897), the expressions of which were confirmed by qRT-PCR, were selected for subsequent analysis. CircRNA-miRNA-mRNA interaction networks were constructed for circRNA_26852 and its target genes as well as circRNA_11897 and its target genes. GO and KEGG enrichment analyses reveal that the target genes of circRNA_26852 and circRNA_11897 are enriched in pathways related to adipocyte differentiation and lipid metabolism, as well as in disease-related pathways. Conclusions: In this study, circRNA sequencing and bioinformatics technique were used to analyze, for the first time, the expression of circRNAs in the subcutaneous adipose tissues of Large White pig and Laiwu pig. It is inferred that circRNAs might regulate adipogenic differentiation and lipid metabolism. The results provide a theoretical basis for further study on fat deposition mechanism and provide potential therapy targets for metabolism-related diseases.
\end{abstract}

(C) 2018 The Author(s)

Published by S. Karger AG, Basel

\section{Introduction}

Adipose tissue is a type of highly specialized loose connective tissue, which stores a high content of triglycerides and lipid-soluble substances. Surplus energy produced by the 
body during metabolism is generally stored in adipose tissue. If the capacity of adipose tissue to absorb and store lipids decreases, lipids may be then accumulated in non-adipose tissues, thus leading to obesity and imbalance in energy metabolism, and further leading to obesity-related diseases such as type II diabetes, atherosclerosis, insulin resistance, nonalcoholic fatty hepatitis, cardiovascular diseases, etc $[1,2] .$. In fact, health issues related to lipid metabolism disorders have received extensive attention, but the specific molecular mechanism remains unclear.

Circular RNA (circRNA) is a kind of endogenous circular molecule generated by alternative splicing. It forms a covalently closed continuous loop without 5' and 3' ends, thus it cannot be degraded by ribonuclease. In mammalian cells, circRNAs exist in large amounts and have properties such as good stability, conservativity and tissue specificity. It has been shown that circRNAs are related to Alzheimer's disease [3], colorectal cancer and ovarian cancer [4], hepatocellular carcinoma [5], cardiovascular diseases [6], central nervous system disorders [7], etc., but their role in lipid metabolism remains unclear.

Recent research suggests that in eukaryotic cells circRNAs can work as miRNA sponges to regulate gene expression $[8,9]$. Currently, there are evidences showing that miRNA can regulate the expression of genes associated with adipogenic differentiation and lipid metabolism [10, 11]. For example, in human preadipocytes and mouse 3T3-L1 cells, miR143 promotes adipocyte differentiation by targeting MAPK1 [12]. Chen et al. reported that overexpression of miR-425-5p would inhibit the proliferation and differentiation of pig intramuscular preadipocytes and lead to down-regulated expression of PPAR $\gamma$, FABP4 and FASN associated with fat formation [13]. In high fat-induced obese mice, miR-199a-3p regulates adipocyte differentiation by directly targeting 3'UTR of SCD, and it can change fatty acid composition by decreasing the proportion of unsaturated fatty acids [14]. However, it remains unknown whether these miRNAs involved in lipid metabolism are regulated by circRNAs.

Pigs are anatomically and physiologically similar to humans. Particularly, pigs and humans have similar cardiovascular, urinary, dermatological and digestive systems, and brown fat disappears in both after birth [15]. Therefore, pig, as an animal model of disease, has important clinical values. Large White pig is a typical lean meat breed with lower contents of subcutaneous fat and intramuscular fat than Laiwu pig. Because of the difference in fat deposition between the two pig breeds, circRNAs in their subcutaneous adipose tissues were sequenced in this paper and the expression patterns of the circRNAs were investigated. Bioinformatics methods were also used to systematically analyze circRNAs that can regulate lipid metabolism. The results may provide a theoretical basis for further study on lipid metabolism, and provide potential therapy targets for lipid metabolism-related diseases.

\section{Materials and Methods}

\section{Ethics statement}

All procedures involving animals were approved by the Animal Care and Use Committee at Institute of Animal Sciences (Chinese Academy of Agricultural Sciences), where the experiments were conducted. All experiments were performed in accordance with the relevant guidelines and regulations set by the Ministry of Agriculture of the People's Republic of China.

\section{Experimental animals and sample preparation}

Experimental animals include two pig breeds: Large White pig and Laiwu pig (a local breed) with difference in fat deposition. Three female pigs of each breed were used. All pigs were raised under the same conditions in the same environment in Daqian Agriculture and Animal Husbandry Co., Ltd. (Laiwu City, China). They were fed according to the current nutritional needs (National Research Council, NRC, 1998). At slaughter age, their body weights were similar within species, and they were all healthy and in good body condition. After slaughter, the subcutaneous adipose tissue of the longissimus dorsi muscle of each animal was sampled. 


\section{Cellular Physiology Cell Physiol Biochem 2018;47:2458-2470 \begin{tabular}{c|l} 
and Biochemistry Published online: July 10, 2018 & $\begin{array}{l}\text { () 2018 The Author(s). Published by S. Karger AG, Basel } \\
\text { www.karger.com/cpb }\end{array}$ \\
\hline
\end{tabular}}

\section{RNA isolation and quality control}

Total RNAs from each sample were isolated with TRIzol reagent (Invitrogen Life Technologies, Carlsbad, USA) according to the manufacturer's instructions. The purity and concentration of the total RNAs were determined with NanoDrop ND-1000. RNA integrity was tested by denaturing agarose gel electrophoresis.

\section{Construction of RNA library and circRNA sequencing}

Sequencing libraries were generated using a TruSeq Stranded Total RNA LT Kit with Ribo-Zero TM Gold (RS-122-2301) for Illumina according to the manufacturer's recommendations. Ribo-Zero rRNA Removal Kit was used to remove ribosomal RNAs from the total extracted RNAs. RNase R was used to remove linear RNAs. Then, breaking reagent was added to break circRNAs into fragments. With the circRNA fragments as templates, random hexanucleotide primers were used to synthesize first-strand cDNAs. Subsequently, second-strand cDNAs were synthesized with second-strand cDNA synthesis mix. When second-strand cDNAs were synthesized, dUTP was used instead of dTTP and different adapters were ligated to the $5^{\prime}$ and $3^{\prime}$ ends of the second strand (The Index Primer were shown in Table 1). UNG enzyme was used to digest the cDNA strand containing dUTP, and only the cDNA strand with different adapters was kept. cDNAs were purified using cDNA purification kit. After purification, end repair was performed, poly (A) tail was added to cDNA and sequencing adapter was ligated. Subsequently, size selection was performed. Then, PCR amplification was performed. The quality of the library was examined with Agilent 2100 Bioanalyzer. After ensuring the quality of the library, Illumina HiSeqTM 2500 (or other sequencing equipment) was used for sequencing.

\section{CircRNA sequencing analysis}

Paired-end sequencing was adopted. After the sequencing data were obtained, NGSQCToolkit (v2.3.3) was used for quality control and adapter trimming [16]. On this basis, low-quality bases and N-bases were trimmed from the reads. Finally, high-quality clean reads were obtained for subsequent analysis. BWA[17] was used to align the clean reads to reference gene sequences. CIRI was used to identify circRNAs. The alignment results (SAM format) were scanned to search for paired chiastic clipping (PCC) and paired-end mapping (PEM) signals, as well as GT-AG splicing signals. All the sequences with junction sites were realigned to reference gene sequences using dynamic programming algorithm to ensure the reliability of the identified circRNAs. DEseq2[18] was used for differential expression analysis of circRNAs. Comparing treatment group and reference group, |fold Change $\geq 2$ and $p$-value $<0.05$ were used to indicate significant difference in circRNA expression. Then, the number of up/down-regulated circRNAs was obtained.

\section{GO and KEGG pathway enrichment analysis}

Gene ontology (GO) (http://www.geneontology.org/) is an international standard for gene function classification. It classifies gene functions into three categories including molecular function, biological process and cellular component. Pathway enrichment analysis can help determine the main metabolic pathways and signaling pathways in which differentially expressed genes are involved. Kyoto Encyclopedia of Genes and Genomes (KEGG, http://www.genome.jp/kegg) database [19] is a main public database for metabolic analysis and regulatory network research. To further explore the main biological functions of differentially expressed genes, we determined the GO terms and signaling pathways in which differentially expressed genes were enriched on the basis of hypergeometric distribution. If q-value $\leq 0.05$, the enrichment was significant.

\section{Real-time fluorescence quantitative PCR verification}

Total RNAs were reverse transcribed to synthesize cDNAs using PrimeScript RT Reagent Kit (Perfect Real Time; TaKaRa, Osaka, Japan), which were then subject to quantitative real-time PCR (qRT-PCR) analysis on an Applied Biosystems 7500 Fast Real-Time PCR System (Roche, Basel, Switzerland; v 2.0.5) with SYBR Green qPCR SMix (ROX; Roche). qRT-PCR results were used for verifying the gene expression levels revealed by above sequencing analysis. Six circRNAs (circRNA_26852, circRNA_15067, circRNA_25555, circRNA_11897, circRNA_23437 and circRNA_14707), three miRNAs (ssc-miR-486, ssc-miR27a and ssc-miR-27b-3p) and three mRNAs (ABHD5, SMAD7 and SCD) were selected for amplification by specific divergent primers (Table 2). MiRNA reverse

Table 1. The index primers used for the circRNA-seq library preparation

\begin{tabular}{cc}
\hline Sample & Index primer \\
\hline D-PX-1 & TGACCA \\
D-PX-2 & ACAGTG \\
D-PX-3 & GCCAAT \\
L-PX-1 & TAGCTT \\
L-PX-2 & GGCTAC \\
L-PX-3 & CTTGTA \\
\hline
\end{tabular}




\section{Cellular Physiology Cell Physiol Biochem 2018;47:2458-2470 and Biochemistry Published online: July 10, $2018 \quad \begin{aligned} & \text { DOI: 10.1159/000491619 } 2018 \text { The Author(s). Published by S. Karger AG, Basel } \\ & \text { www.karger.com/cpb }\end{aligned}$ \\ Li et al.: Identification and Characterization of CircRNAs in Pigs}

primers are universal primers that come with the QIAGEN kit. With glyceraldehyde3-phosphate dehydrogenase (GAPDH) gene, $5 \mathrm{~S}$, Beta-actin (ACTB) as internal references of circRNA, miRNA, mRNA, respectively, $2^{-\Delta \Delta \mathrm{ct}}$ method was used to calculate the relative expression levels of gene between samples. t-test was performed to statistically analyze the relative expression levels.

Bioinformatics analysis and target gene prediction

miRanda software [20] was used to perform miRNA target prediction for circRNAs. In combination with our previous results on miRNAs and mRNAs in subcutaneous adipose tissues of Large White pig and Laiwu pig [21, 22], miRanda [20] and Targetscan [23] were used to predict the target genes of differentially expressed miRNAs. Differentially expressed mRNAs among miRNA target genes were selected and Cytoscape [24] was used to construct the circRNA-miRNA-mRNA interaction network. ClueGO[25] and KOBAS[26] were used to perform GO and KEGG enrichment analyses, respectively, of the differentially expressed genes related to the differentially expressed circRNAs.

Statistical analysis

All data were presented as mean values \pm standard deviations. When comparisons were made, a Student's t-test was performed. $\mathrm{p}<0.05$ was considered as statistically significant.

\section{Results}

Differential expression analysis of circRNAs

Laiwu pig and Large White pig have difference in fat deposition. By comparing the expression of circRNAs between two pig breeds, we identified circRNAs that might potentially regulate fat deposition in them and further explored the underlying molecular mechanism. A total of 29, 763 circRNAs were identified in the subcutaneous adipose tissues of Laiwu pig and Large White pig. Differential expression analysis was performed (Laiwu pig vs. Large White pig, denoted as L_PX vs. D_PX). With |fold change $\mid \geq 2.0$ and p-value $<0.05$ as criterion (Fig. 1), 275 differentially expressed circRNAs (70 up-regulated circRNAs and 205 downregulated circRNAs) were obtained. The up-regulated circRNAs included 3 exonic circRNAs, 17 intergenic circRNAs, 49 sense-overlapping circRNAs and 1 antisense circRNAs (Fig. 2A), while the down-regulated circRNAs included 7 exonic circRNAs, 21 intergenic circRNAs, 174 sense-overlapping circRNAs and 3 antisense circRNAs (Fig. 2B).

\section{qRT-PCR verification}

According to our previous results on miRNAs and mRNAs in subcutaneous adipose tissues of Large White pig and Laiwu pig $[21,22]$ as well as the target miRNAs of circRNAs, six differentially expressed circRNAs were further verified by qRT-PCR. The results show that in subcutaneous adipose tissue of Laiwu pig, circRNA_26852, ssc-miR-486 and SCD were significantly up-regulated, while circRNA_15067, circRNA_23437, circRNA_14707, circRNA_11897, ssc-miR-27a, ssc-miR-27b-3p, ABHD5 and SMAD7 were significantly down-regulated. circRNA_25555 tended to be down-regulated but the difference was not statistically significant (Fig. 3).

CircRNA-miRNA target prediction

CircRNAs can work as miRNA sponges and regulate miRNAs. Analysis of circRNAmiRNA interaction can help reveal the functions and functioning mechanisms of circRNAs 
as miRNA sponges. miRNA-circRNA interaction pairs were sorted according to p-values and the top 300 miRNA-circRNA interaction pairs with smaller p-values were selected for the construction of circRNA-miRNA interaction network (Fig. 4).

\section{CircRNA-miRNA-mRNA interaction network}

The expression levels of circRNAs determined by qRT-PCR were consistent with those determined by sequencing analysis. circRNA_26852 and circRNA_11897 that were upregulated and down-regulated, respectively, in the subcutaneous adipose tissue of Laiwu pig were selected for subsequent analysis. ssc-miR-874 and ssc-miR-486 that can bind circRNA_26852 as well as ssc-miR-27a and ssc-miR-27b-3p that can bind circRNA_11897

Fig. 1. The distribution of differentially expressed circRNAs between two pig breeds. Red points represent upregulated circRNAs, and green points represent down-regulated circRNAs. Gray points represent circRNAs without significant difference in expression between two pig breeds ( fold change $\mid<2.0$, p-value $\geq 0.05$ ). Blue points represent circRNAs without significant difference in expression between two pig breeds (|fold change $\mid \geq 2.0$, but $\mathrm{p}$-value $\geq 0.05$ ).
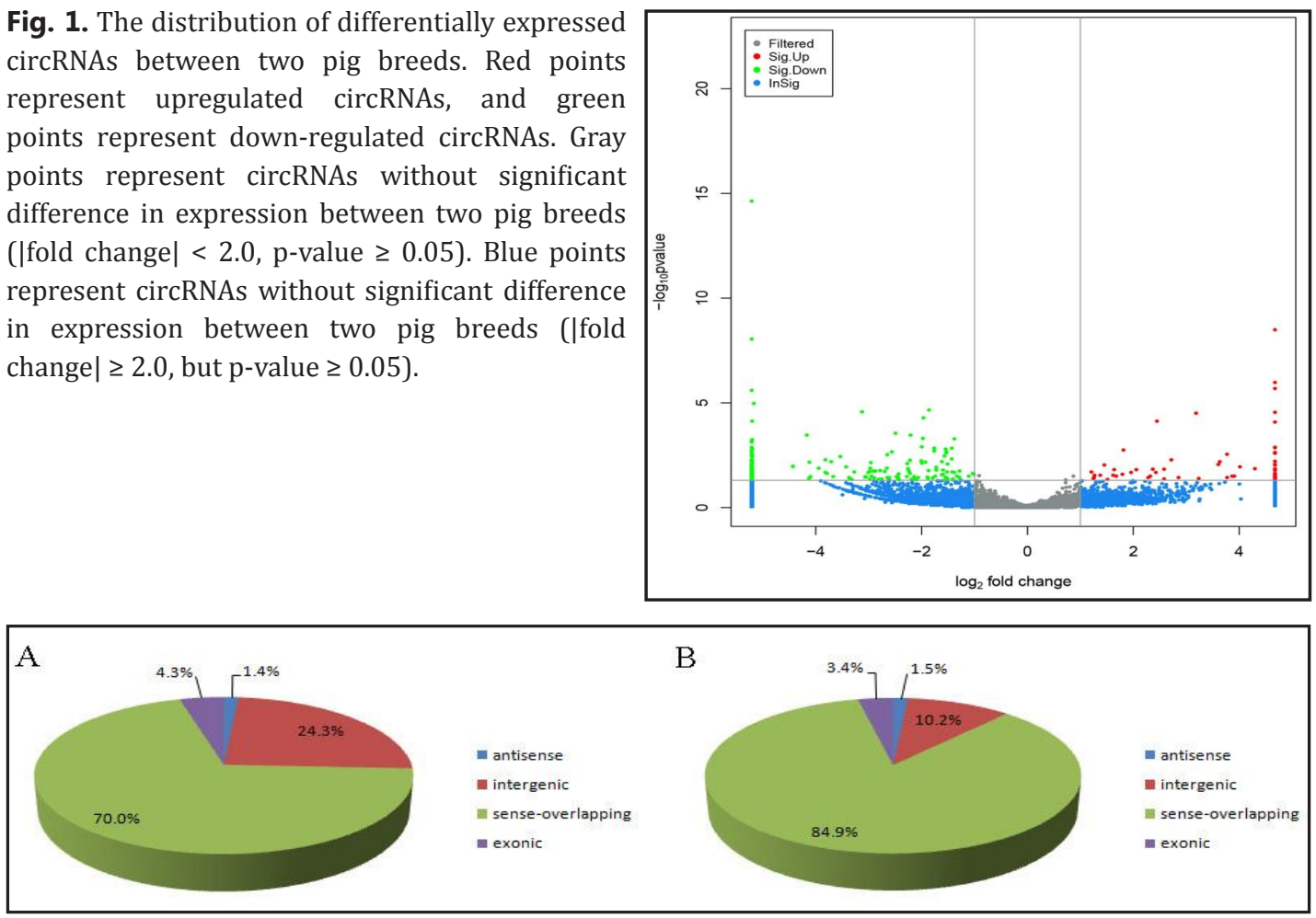

Fig. 2. The proportion of various circRNAs in total differentially expressed circRNAs in subcutaneous adipose tissue of Laiwu pig. (A) Up-regulated circRNAs. (B) Down-regulated circRNAs.

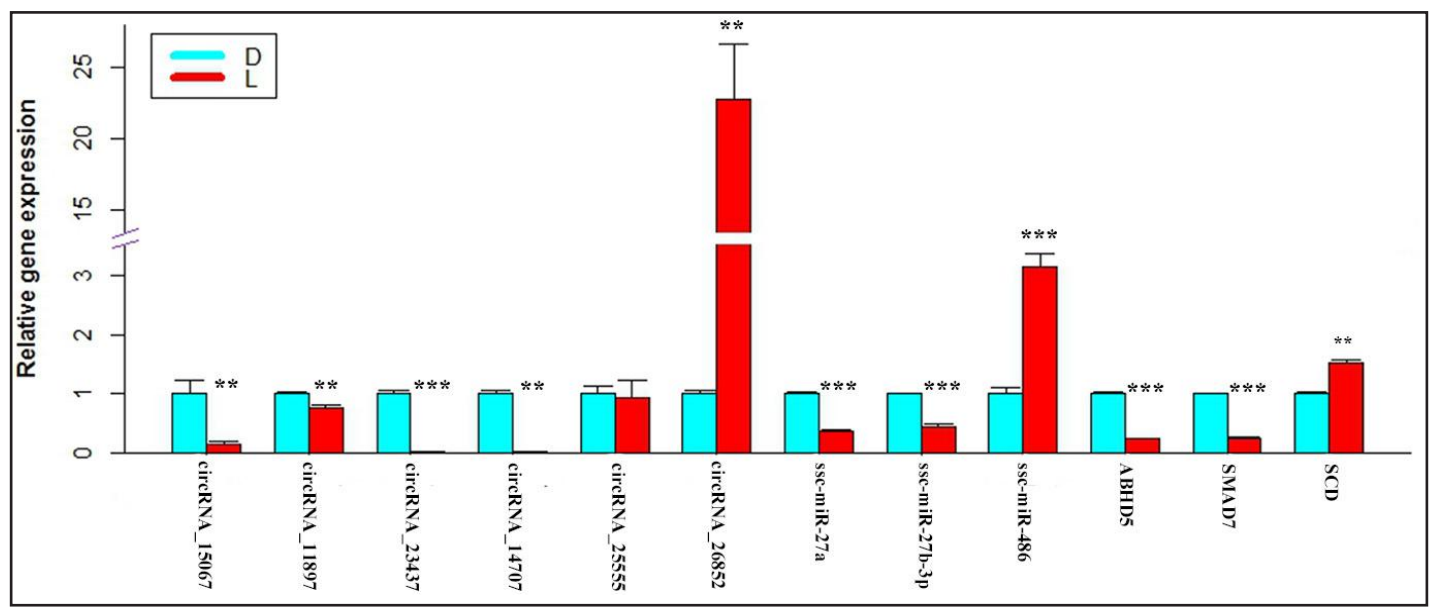

Fig. 3. qRT-PCR validation of the differentially expressed circRNAs, miRNAs and mRNAs. *: P<0.05; **: $\mathrm{P}<0.01 ; * * *: \mathrm{P}<0.001$. 
(Table 3) were selected according to bioinformatics analysis and our previous results [21, 22] on miRNAs and mRNAs in the subcutaneous adipose tissue of two pig breeds. Subsequently, circRNA-miRNA-mRNA interaction networks were constructed (Fig. 5). Four miRNAs (sscmiR-874, ssc-miR-486, ssc-miR-27a, and ssc-miR-27b-3p) in Fig. 5 were differentially expressed in the subcutaneous adipose tissues of Large White pig and Laiwu pig (Table 4). Other miRNAs in Fig. 5 were not differentially expressed but might also be potential targets of circRNA_26852 and circRNA_11897. All the mRNAs in Fig. 5 were differentially expressed in the subcutaneous adipose tissues of Large White pig and Laiwu pig [21]. qRT-PCR results of some genes including SCD, ABHD5 and SMAD7 were consistent with our previous sequencing results of differentially expressed mRNAs.

\section{GO and KEGG enrichment analyses of differentially expressed target genes}

In order to deeply explore the functions of targetgenes, GO and KEGG pathway enrichment analyses were performed. The target genes of circRNA_26852 are mainly enriched in biological processes related to fat deposition and lipid metabolism, such as regulation of protein kinase D signaling, regulation of triglyceride catabolic process, negative regulation of lipid storage, phosphatidic acid biosynthetic process, etc. (Fig. 6A). Pathway enrichment analysis indicates that the target genes of circRNA_26852 are enriched in biosynthesis of unsaturated fatty acids, fatty acid metabolism, PPAR signaling pathway, TGF-beta signaling pathway, and disease-related signaling pathways (such as Insulin signaling pathway, AGE-

Table 3. circRNA-miRNA interactions in the subcutaneous adipose tissue of Laiwu pig. Note: Position presents the start site that miRNA binds to circRNA region

\begin{tabular}{|c|c|c|c|c|c|}
\hline miRNA & circRNA & $\begin{array}{c}\text { Max } \\
\text { score }\end{array}$ & $\begin{array}{c}\text { Min } \\
\text { energy }\end{array}$ & $\begin{array}{l}\text { miRNA } \\
\text { length }\end{array}$ & Postion \\
\hline ssc-miR-874 & $\begin{array}{c}\text { circRNA_26852 } \\
\text { (chr9:50919101_50997349_+) }\end{array}$ & 164 & -32.99 & 23 & $\begin{array}{c}30424306632592 \\
281\end{array}$ \\
\hline ssc-miR-486 & $\begin{array}{c}\text { circRNA_26852 } \\
\text { (chr9:50919101_50997349_+) }\end{array}$ & 170 & -31.83 & 22 & 16171 \\
\hline $\begin{array}{l}\text { ssc-miR-27b- } \\
3 p\end{array}$ & $\begin{array}{c}\text { circRNA_11897 } \\
\left(\operatorname{chr} 15: 107266814 \_107322566 \_+\right)\end{array}$ & 191 & -33.04 & 21 & 32819 \\
\hline ssc-miR-27a & 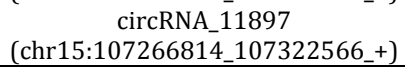 & 186 & -31.28 & 21 & 32819 \\
\hline
\end{tabular}

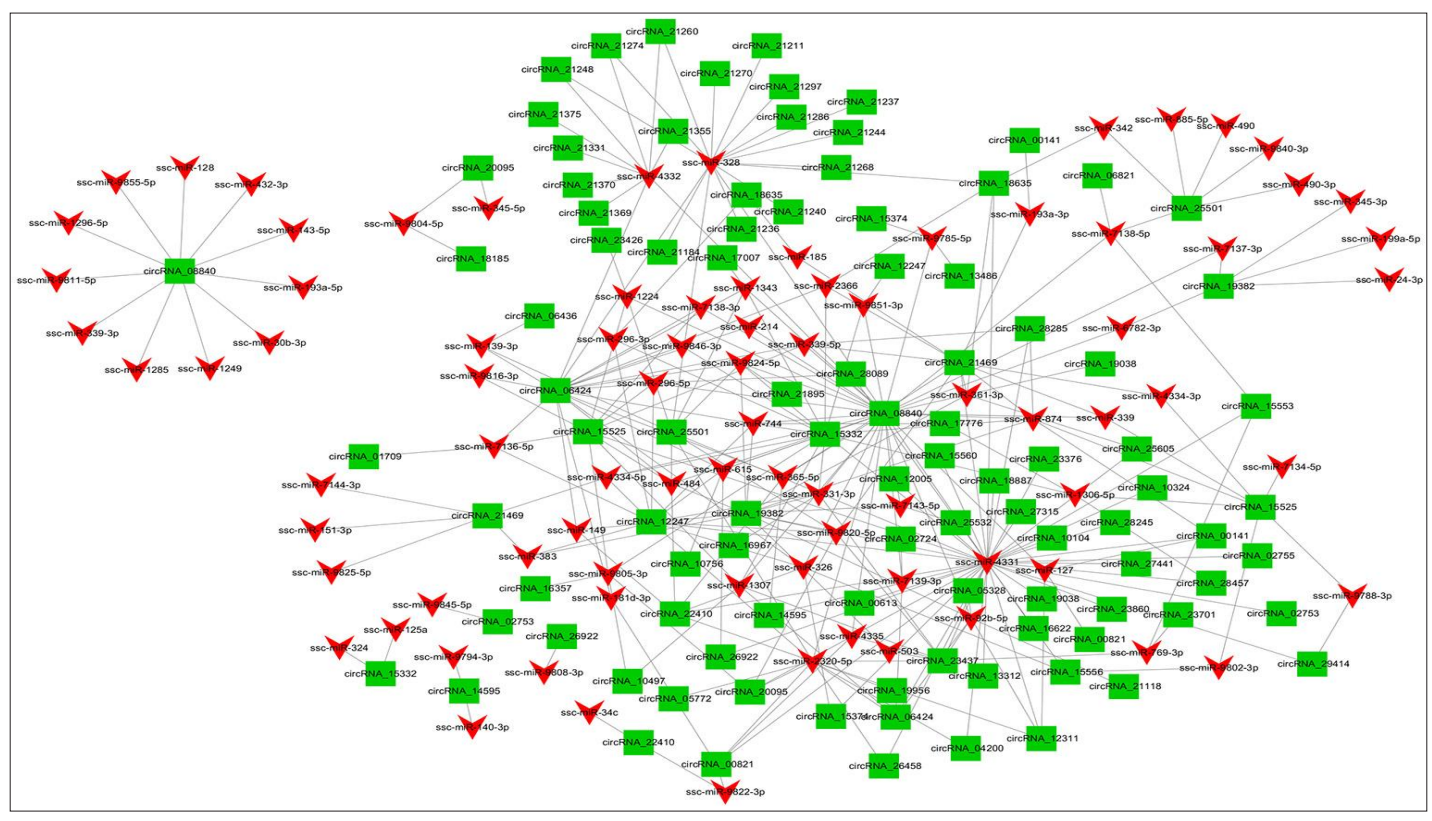

Fig. 4. Co-expression network of the miRNAs and differentially expressed circRNAs in subcutaneous adipose tissue between two pig breeds. Red represents miRNAs and green represents circRNAs. 
RAGE signaling pathway in diabetic complications, Huntington's disease, etc.) (Fig. 6B). The target genes of circRNA_11897 are also enriched in biological processes related to lipid metabolism such as fatty acid biosynthetic process, MAPK cascade reaction, ERK1 and ERK2 cascade reaction, cell proliferation, etc. (Fig. 7A). Pathway enrichment analysis shows that the target genes of circRNA_11897 are enriched in PPAR signaling pathway, AMPK signaling pathway, non-alcoholic fatty liver disease (NAFLD), Alzheimer's disease, pathways in cancer, etc. (Fig. 7B). Differentially expressed miRNAs might target these differentially expressed genes in the pathways and then change their signal transduction. Since circRNAs can work as miRNA sponges, circRNAs might inhibit the effect of miRNAs on the target genes and then regulate fat deposition and metabolism-related diseases.

\section{Discussion}

This study analyzed, for the first time, the expression of circRNAs in the subcutaneous adipose tissues of Large White pig and Laiwu pig. The aim was to identify potential circRNAs related to adipogenic differentiation and lipid metabolism. A total of 275 differentially expressed circRNAs were identified in the subcutaneous adipose tissue. Among them, 70 were up-regulated and 205 were down-regulated in the subcutaneous adipose tissue of Laiwu pig. The expressions of six circRNAs were further validated by qRT-PCR analysis.

Table 4. Differentially expressed miRNAs in the subcutaneous adipose tissues of Large White pig and Laiwu pig

\begin{tabular}{lccccc}
\hline miR_name & Mean (D) & Mean (L) & $\begin{array}{c}\text { Log2(foldchange) } \\
\text { (L/D) }\end{array}$ & p_value & q_value \\
\hline ssc-miR-874 & 5.260857 & 10.53275 & 1.001512014 & 0.000163292 & 0.004652 \\
ssc-miR-486 & 19.28981 & 387.6535 & 4.328856993 & 0 & 0 \\
ssc-miR-27b-3p & 5127.699 & 2329.432 & -1.13833331 & 0 & 0 \\
ssc-miR-27a & 314.5993 & 149.7587 & -1.07087528 & $3.58 E-108$ & $4.14 \mathrm{E}-106$ \\
\hline
\end{tabular}

Fig. 5. circRNA-miRNAmRNA interaction networks. (A) circRNA_26852 with 41 miRNA recognition elements (MREs) and differentially expressed target genes and (B) circRNA_11897 with 10 MREs and differentially expressed target genes. Red square represents up-regulated circRNA_26852 in Laiwu pig adipose tissue. Yellow square represents down-regulated circRNA_11897 in Laiwu pig adipose tissue. Blue arrows represent miRNAs. Green circles represent mRNAs.

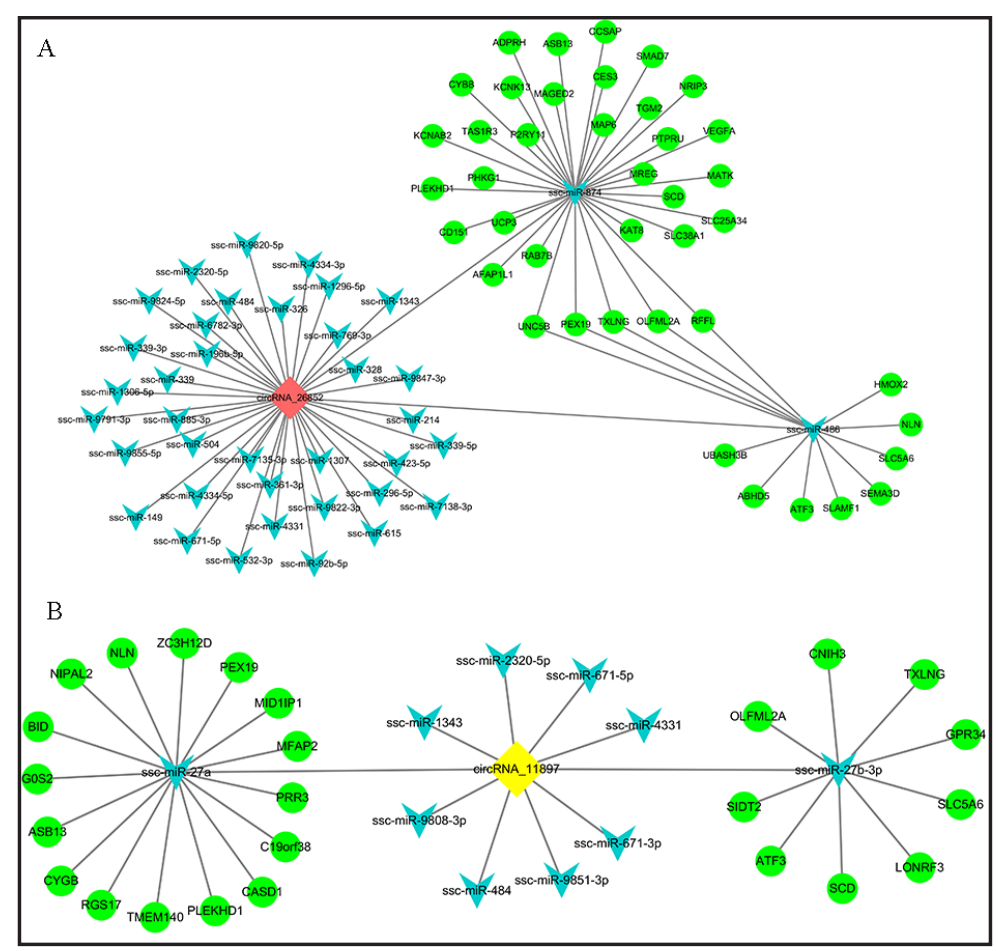


Fig. 6. (A) GO enrichment analysis and (B) KEGG pathway enrichment analysis of the target genes of circRNA_26852. (A) is composed of three parts: biological processes, molecular functions, and cellular components. The significance level of enrichment was set at q-value $<0.05$. In (B), the vertical axis refers to the significantly enriched pathways with q-value $<0.05$.

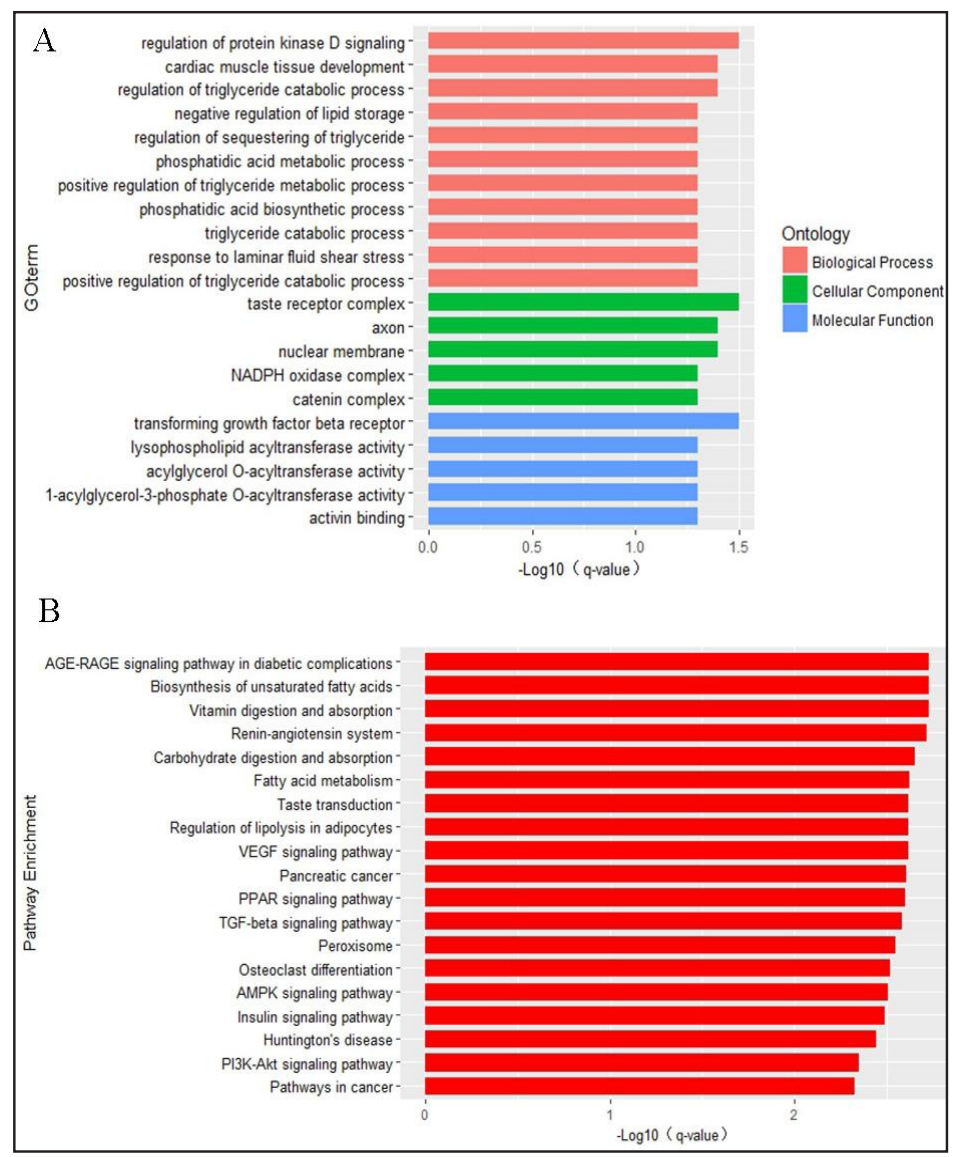

Fig. 7. (A) GO enrichment analysis and (B) KEGG pathway enrichment analysis of the target genes of circRNA_11897. (A) is composed of three parts: biological processes, molecular functions, and cellular components. The significance level of enrichment was set at q-value $<0.05$. In (B), the vertical axis refers to the significantly enriched pathways with q-value $<0.05$.

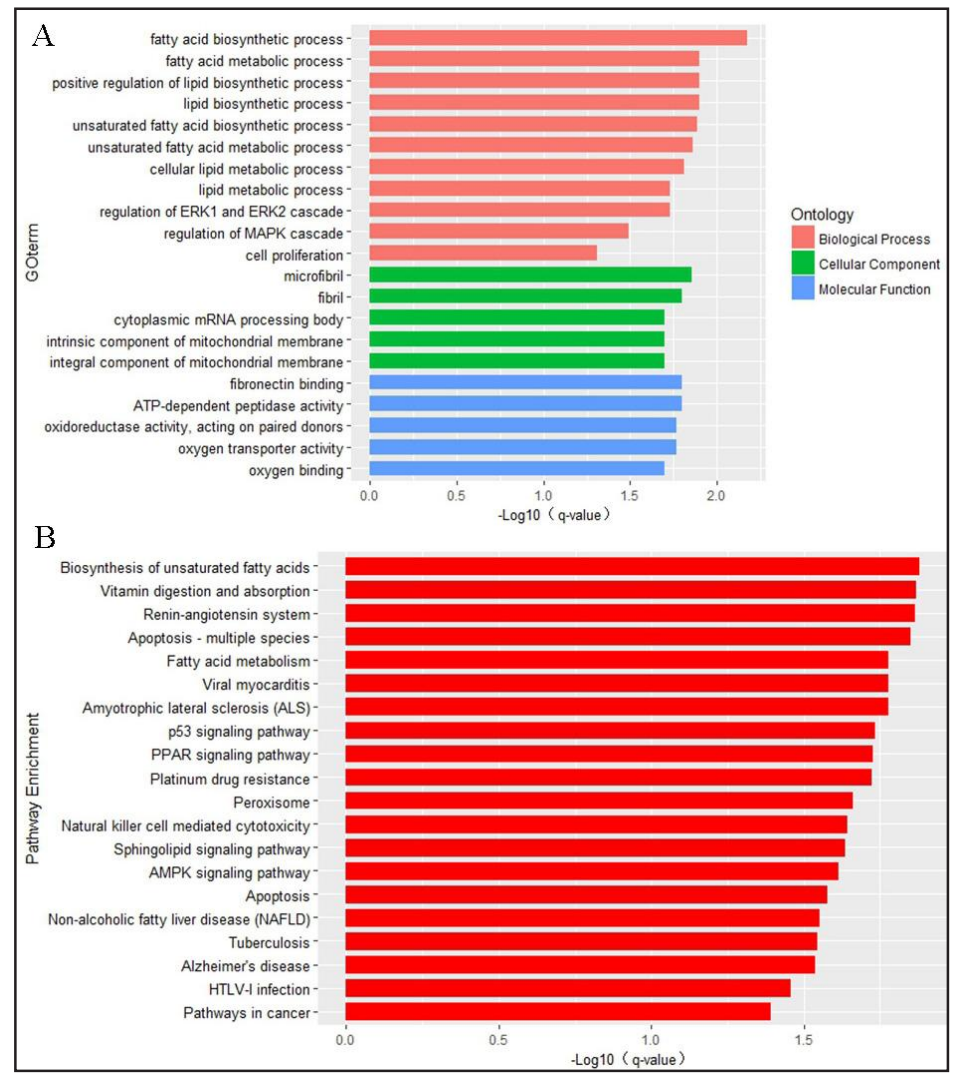


miRNA target prediction was performed for these differentially expressed circRNAs. The host genes of differentially expressed circRNAs and target genes of up-regulated circRNA_26852 and down-regulated circRNA_11897 were subject to GO and KEGG enrichment analysis. The regulatory role of circRNAs in fat deposition and lipid metabolism was explored from the perspective of host genes and competing endogenous RNAs. Our results may provide potential therapy targets for obesity and metabolic syndrome.

CircRNAs can absorb miRNAs associated with adipogenic differentiation and lipid metabolism. For the differentially expressed circRNAs, miRanda was used to detect some differentially expressed miRNAs that can bind them. It was found that ssc-miR-874 and sscmiR-486 are targets of circRNA_26852, and ssc-miR-27a and ssc-miR-27b-3p are targets of circRNA_11897. Guo et al. reported that the expression of miR-874 is down-regulated in the adipose tissue of Japanese Black Wagyu cattle compared with that in Holstein cattle, thus miR-874 might regulate fat deposition in cattle [27]. In the subcutaneous adipose tissue of Laiwu pig, the expression of miR-874 was up-regulated. Japanese Black Wagyu cattle and Laiwu pig both have strong ability to deposit fat in their bodies, but the expression levels of miR-874 in them are different. It is inferred that this might be related to species and location. Some research indicates that in the subcutaneous adipose tissue of HIV-infected patients the expression of some miRNAs is abnormal and among them the expression of miR-874 is upregulated [28]. Then, the research infers that changes in the expression levels of these miRNAs might lead to lipid metabolism disorders related to HIV. In addition, miR-874 might promote insulin synthesis [29]. Therefore, it is inferred that miR-874 is related to fat deposition and fat metabolism. Prats-Puig et al. found that the expression of miR-486 is improved in the plasma of obese children before adolescence and it is related to fat content, adiponectin, etc [30].. The expression of miR-486 in these obese children is consistent with that in Laiwu pig. Some report shows that miR-486 can inhibit the transcription factor, i.e., forkhead box 01 (Fox 01)[31], and plays a role in insulin functioning and triglyceride metabolism [32]. Consequently, it is inferred that miR-486 might be involved in lipid metabolism and associated with diabetes and obesity. Since ssc-miR-874 and ssc-miR-486 are targets of circRNA_26852, circRNA_26852 might participate in adipogenic differentiation and lipid metabolism. Wang et al. found that overexpression of miR-27a in pig adipocytes could promote lipolysis and the production of more glycerin and fatty acids [33]. Some report indicates that miR-27a can inhibit adipocyte differentiation by targeting PPAR $\gamma$ [34]. Song et al. found that miR-27b-3p can regulate fatty acid metabolism by targeting PPAR $\gamma$ and then play a role in the maturation and development of pig oocytes [35]. circRNA_11897 can bind miR-27a and miR-27b-3p, which might lead to the up-regulation of the target genes of miR-27a and miR-27b-3p and then the regulation of adipogenic differentiation and lipid metabolism. In sum, it is inferred that circRNAs may work as competing endogenous RNAs and regulate lipid metabolism, which may provide potential prevention targets for metabolism-related diseases.

Competing endogenous RNAs (ceRNAs) are RNAs with miRNA recognition elements. ceRNAs and mRNAs can competitively bind the same miRNA [36], thereby regulating each other. This mechanism is called "ceRNA" hypothesis. The target genes of circRNAs were analyzed to explore in depth the role of circRNAs in fat deposition and fat metabolism. GO and KEGG enrichment analyses reveal that the target genes of circRNA_26852 are enriched in biological processes related to fat deposition and lipid metabolism such as regulation of triglyceride catabolic process, negative regulation of lipid storage, phosphatidic acid biosynthetic process, etc. Pathway enrichment analysis shows that the target genes of circRNA_26852 are enriched in PPAR signaling pathway, TGF-beta signaling pathway, and disease-related signaling pathways (such as insulin signaling pathway AGE-RAGE signaling pathway in diabetic complications, Huntington's disease). Zhang et al. reported that miR-140$3 p$ can regulate adipocyte differentiation by targeting TGF- $\beta 1$ [37]. In addition, TGF- $\beta 1$ can phosphorylate SMAD3. The activated SMAD3 can bind C/EBPs to inhibit its transcriptional activity and thus play a role in the early stage of adipocyte differentiation [38]. PPARs are members of receptor superfamily and they include three subtypes: PPAR $\alpha$, PPAR $\beta$ and PPAR $\gamma$. PPAR $\alpha$ gene can promote the hydrolysis of triglycerides into free fatty acids, activate

\section{KARGER}




\section{Cellular Physiology Cell Physiol Biochem 2018;47:2458-2470 \\ \begin{tabular}{l|l} 
DOI: 10.1159/000491619 & O 2018 The Author(s). Published by S. Karger AG, Basel \\
www.karger.com/cpb
\end{tabular} \\ Li et al.: Identification and Characterization of CircRNAs in Pigs}

$\beta$ oxidation in liver and muscle, and decrease the synthesis of fatty acids and glycerin [39]. PPAR $\gamma$ is a key transcription factor regulating the transformation of mesenchymal stem cells into adipocytes [40]. The target genes of circRNA_11897 are also enriched in biological processes related to lipid metabolism such as fatty acid biosynthetic process, MAPK cascade reaction, ERK1 and ERK2 cascade reaction, cell proliferation, etc. MAPK, as serine/threonine protein kinase, can phosphorylate many protein kinases and nuclear transcription factors. MAPK cascade reaction is thus one of the important signaling pathways in cell. Four MAPK signaling pathways have been identified in mammalian cells: p38 MAPK pathway, ERK1/2 signaling pathway, ERK5/BMK1 pathway and JNK pathway. Zhao et al. found that activated p38 MAPK can promote fat synthesis in bone marrow mesenchymal stem cells [41]. In early stage of adipogenic differentiation, activated ERK signaling pathway can promote the expression of C/EBP and PPAR $\gamma$ and promote adipocyte differentiation [42]. In later stage, ERK1/2 phosphorylates and inactivate PPAR $\gamma$, thus adipocyte differentiation is inhibited [43]. Therefore, the regulatory role of ERK1/2 in adipocyte differentiation is determined by the accurate time of ERK phosphorylation [44]. It is thus inferred that circRNA might regulate the expression of related genes in signaling pathway by binding miRNA. In this way, circRNA plays a regulatory role in fat deposition and lipid metabolism.

Analysis of circRNA-miRNA-mRNA interaction network shows that separating the synthetic ligands of $\alpha$ - $\beta$-hydrolase domain containing protein 5 (ABHD5, target gene of sscmiR-486) from perilipin-1 (PLIN1) can directly activate adipose triglyceride lipase (ATGL) and promote lipolysis [45]. Our previous study shows that the expression of ABHD5 is downregulated in the subcutaneous adipose tissue of Laiwu pig [21], whereas the expression of ssc-miR-486 is up-regulated in the subcutaneous adipose tissue of Laiwu pig [22]. Since circRNA_26852 can work as miR-486 sponge, it is inferred that circRNA_26852-miR-486ABDH5 axis can regulate lipid metabolism. SCD gene is the target gene of ssc-miR-27b-3p. SCD is a key enzyme in lipogenesis and it can catalyze the transformation of saturated fatty acid (SFA) into monounsaturated fatty acid (MUFA) and play an important role in biosynthesis of fatty acid [46]. There is a lack of SCD1 gene in mice, thus feeding the mice with sugar cannot induce the synthesis and secretion of triglycerides [47]. The expression of SCD is upregulated in the subcutaneous adipose tissue of Laiwu pig [21], while the expression of sscmiR-27b-3p is down-regulated [22]. Bioinformatics analysis shows that circRNA_11897 can work as miR-27b-3p sponge. It is inferred that circRNA_11897 might play a role in regulating adipogenic differentiation and lipid metabolism. SMAD is a kind of intracellular protein and it can change extracellular signal into intracellular growth factor receptor, which then activate the transcription of downstream genes in nucleus $[48,49]$. SMAD7 can inhibit the activation of SMAD2 and SMAD3 by TGF $\beta$ [50], and participate in adipocyte differentiation [51]. The expression of SMAD7 gene, as the target gene of ssc-miR-874, is down-regulated in the subcutaneous adipose tissue of Laiwu pig [21], while the expression of ssc-miR-874 is up-regulated [22]. Since circRNA_26852 can work as ssc-miR-874 sponge, it is inferred that circRNA_26852 might regulate the expression of target genes by binding ssc-miR-874 and further regulate adipocyte differentiation and lipid metabolism.

\section{Conclusion}

In sum, this study used circRNA sequencing and bioinformatics technique to analyze, for the first time, the expression of circRNAs in the subcutaneous adipose tissues of Large White pig and Laiwu pig. CircRNA-miRNA target prediction was performed and circRNAmiRNA-mRNA interaction network was constructed. By GO and KEGG pathway analyses, we identified pathways enriched in adipogenic differentiation and lipid metabolism, as well as disease-related pathways. It is inferred that circRNAs might regulate adipogenic differentiation and lipid metabolism. This research provides a theoretical basis for further study on fat deposition mechanism and provides potential therapy targets for metabolismrelated diseases. 


\section{Cellular Physiology Cell Physiol Biochem 2018;47:2458-2470 \begin{tabular}{l|l} 
DOI: 10.1159/000491619 & $\begin{array}{l}\text { O 2018 The Author(s). Published by S. Karger AG, Basel } \\
\text { www.karger.com/cpb }\end{array}$
\end{tabular}}

\section{Acknowledgements}

X.Y.M. conceived and designed the study and wrote the paper. A.L. performed the experiment, analyzed data and wrote the paper. W.L.H., X.X.Z. and L.L.X performed the experiment and interpreted the data. All the authors read and approved the final manuscript. This work was supported by a grant from the Agricultural Science and Technology Innovation Program (ASTIPIAS05), the National Basic Research and Development Program of China (973 Program) (No. 2015CB943100) and the Basic Research Fund for Central Public Research Institutes of CAAS (No. 2013ywf-zd-2).

\section{Disclosure Statement}

The authors declare that they have no competing interests.

\section{References}

1 Wong S, Song K, Srivastava R, Dong C, Go GW, Li N, Iwakiri Y, Mani A: Nonalcoholic fatty liver disease induced by noncanonical Wnt and its rescue by Wnt3a. Faseb J 2015;29:3436-3445.

-2 Nora K, Matthias B: Adipocyte dysfunction, inflammation and metabolic syndrome. Rev Endocr Metab Disord 2014;15:277-287.

-3 Lukiw WJ: Circular RNA (circRNA) in Alzheimer's disease (AD). Front Genet 2013;4:307.

-4 Bachmayr-Heyda A, Reiner AT, Auer K, Sukhbaatar N, Aust S, Bachleitner-Hofmann T, Mesteri I, Grunt TW, Zeillinger R, Pils D: Correlation of circular RNA abundance with proliferation --exemplified with colorectal and ovarian cancer, idiopathic lung fibrosis, and normal human tissues. Sci Rep 2015;5:8057.

5 Qin M, Liu G, Huo X, Tao X, Sun X, Yang J, Fan J, Liu L, Qin W: Hsa_circ_0001649: A circular RNA and potential novel biomarker for hepatocellular carcinoma. Cancer Biomark 2016;16:161-169.

-6 Jiang J, Yang Y, Jiang R: Regulating mechanisms of circRNA and their relationship with cardiovascular diseases. Zhonghua Xin Xue Guan Bing Za Zhi 2016;44:364-366.

7 Lu D, Xu AD: Mini Review: Circular RNAs as Potential Clinical Biomarkers for Disorders in the Central Nervous System. Front Genet 2016;7:53.

8 Hansen TB, Jensen TI, Clausen BH, Bramsen JB, Finsen B, Damgaard CK, Kjems J: Natural RNA circles function as efficient microRNA sponges. Nature 2013;495:384-388.

-9 Hansen TB, Kjems J, Damgaard CK: Circular RNA and miR-7 in cancer. Cancer Res 2013;73:5609-5612.

10 Miao XY, Luo QM, Qin XY: Genome-wide analysis reveals the differential regulations of mRNAs and miRNAs in Dorset and Small Tail Han sheep muscles. Gene 2015;562:188-196.

11 Miao XY, Luo QM, Qin XY, Guo YT: Genome-wide analysis of microRNAs identifies the lipid metabolism pathway to be a defining factor in adipose tissue from different sheep. Sci Rep 2015;5:18470.

$\checkmark 12$ Takanabe R, Ono K, Abe Y, Takaya T, Horie T, Wada H, Kita T, Satoh N, Shimatsu A, Hasegawa K: Up-regulated expression of microRNA-143 in association with obesity in adipose tissue. Biochem Biophys Res Commun 2008;376:728-732.

13 Chen FF, Xiong Y, Peng Y, Gao Y, Qin J, Chu GY, Pang WJ, Yang GS: miR-425-5p Inhibits Differentiation and Proliferation in Porcine Intramuscular Preadipocytes. Int J Mol Sci 2017;18:2101.

14 Tan Z, Du J, Shen L, Liu C, Ma J, Bai L, Jang Y, Tang G, Li M, Li X, Zhang S, Zhu L: miR-199a-3p affects adipocytes differentiation and fatty acid composition through targeting SCD. Biochem Biophys Res Commun 2017;492:82-88.

15 Spurlock ME, Gabler NK: The development of porcine models of obesity and the metabolic syndrome. J Nutr 2008;138:397-402.

16 Pater RK, Jain M: NGS QC Toolkit: a toolkit for quality control of next generation sequencing data. PLoS One 2012; 7:e30619.

17 Li H, Durbin R: Fast and accurate long-read alignment with Burrows-Wheeler transform. Bioinformatics 2010;26:589-595. 


\section{Cellular Physiology Cell Physiol Biochem 2018;47:2458-2470 \begin{tabular}{l|l} 
DOI: 10.1159/000491619 & Ond Biochemistry 2018 The Author(s). Published by S. Karger AG, Basel \\
www.karger.com/cpb
\end{tabular}

18 Love MI, Huber W, Anders S: Moderated estimation of fold change and dispersion for RNA-seq data with DESeq2. Genome Biol 2014;15:550.

19 Kanehisa M, Sato Y, Kawashima M, Furumichi M, Tanabe M: KEGG as a reference resource for gene and protein annotation. Nucleic Acids Res 2016;44:D457-462.

-20 Pasquinelli AE: MicroRNAs and their targets: recognition, regulation and an emerging reciprocal relationship. Nat Rev Genet 2012;13:271-282.

-21 Huang WL, Zhang XX, Li A, Xie LL, Miao XY: Differential regulation of mRNAs and lncRNAs related to lipid metabolism in two pig breeds. Oncotarget 2017;8:87539-87553.

-22 Zhang XX, Huang WL, Guo YT, Miao XY: An integrated analysis of microRNAs involved in fat deposition in different pig breeds. Oncotarget DOI: 10.18632 /oncotarget.22834.

23 Enright AJ, John B, Gaul U, Tuschl T, Sander C, Marks DS: MicroRNA targets in Drosophila. Genome Biol 2003;5:R1. DOI: 10.1186/gb-2003-5-1-r1.

-24 Smoot ME, Ono K, Ruscheinski J, Wang PL, Ideker T: Cytoscape 2.8: new features for data integration and network visualization. Bioinformatics 2011;27:431-432.

-25 Bindea G, Mlecnik B, Hackl H, Charoentong P, Tosolini M, Kirilovsky A, Fridman WH, Pages F, Trajanoski Z, Galon J: ClueGO: a Cytoscape plug-in to decipher functionally grouped gene ontology and pathway annotation networks. Bioinformatics 2009;25:1091-1093.

-26 Xie C, Mao XZ, Huang JJ, Ding Y, Wu JM, Dong S, Kong L, Gao G, Li CY, Wei LP: KOBAS 2.0: a web server for annotation and identification of enriched pathways. Nucleic Acids Res 2011;39:W316-322.

27 Guo YT, Zhang XX, Huang WL, Miao XY: Identification and characterization of differentially expressed miRNAs in subcutaneous adipose between Wagyu and Holstein cattle. Sci Rep 2017;7:44026.

28 Squillace N, Bresciani E, Torsello A, Bandera A, Sabbatini F, Giovannetti C, Giunta G, Rovati L, Del BM, Locatelli V, Gori A: Changes in subcutaneous adipose tissue microRNA expression in HIV-infected patients. J Antimicrob Chemother 2014;69:3067-3075.

29 Zhang Q Xiao XH, Li M, Li WH, Yu M, Zhang HB, Ping F, Wang ZX, Zheng J, Xiang HD: miR-375 and miR-30d in the effect of chromium-containing Chinese medicine. J Diabetes Res 2014;2014:862473.

- 30 Prats-Puig A, Ortega FJ, Mercader-Navarrete JM, Moreno M, Moreno JM, Bonet N, Ricart W, López-Bermejo A, Fernández-Real JM: Changes in circulating microRNAs are associated with childhood obesity. J Clin Endocrinol Metab 2013;98:E1655-1660.

31 Xu J, Li RS, Workeneh B, Dong YL, Wang XN, Hu ZY: Transcription factor FoxO1, the dominant mediator of muscle wasting in chronic. Kidney Int 2012;82:401-411.

- 32 Altomonte J, Cong L, Harbaran S, Richter A, Xu J, Meseck M, Dong HH: Foxo1 mediates insulin action on apoC-III and triglyceride metabolism. J Clin Invest 2004;114:1493-1503.

-33 Wang T, Li MZ, Guan JQ Li PH, Wang HY, Guo YQ, Shuai SR, Li XW: MicroRNAs miR-27a and miR-143 regulate porcine adipocyte lipid metabolism. Int J Mol Sci 2011;12:7950-7959.

-34 Kim SY, Kim AY, Lee HW, Son YH, Lee GY, Lee JW, Lee YS, Kim JB: miR-27a is a negative regulator of adipocyte differentiation via suppressing PPARgamma expression. Biochem Biophys Res Commun 2010;392:323-328.

-35 Song C, Yao J, Cao C, Liang X, Huang J, Han Z, Zhang Y, Qin G, Tao C, Li C, Yang H, Zhao J, Li K, Wang Y: PPARgamma is regulated by miR-27b-3p negatively and plays an important role in porcine oocyte maturation. Biochem Biophys Res Commun 2016;479:224-230.

-36 Chen YH, Li C, Tan CL, Liu XB: Circular RNAs: a new frontier in the study of human diseases. J Med Genet 2016;53:359-365.

-37 Zhang X, Chang AL, Li YM, Gao YF, Wang HX, Ma ZS, Li XX, Wang BL: miR-140-5p regulates adipocyte differentiation by targeting transforming growth factor- $\beta$ signaling. Sci Rep 2015;5:18118.

-38 Song WW, McLennan SV, Tam C, Williams PF, Baxter RC, Twigg SM: CCN2 requires TGF-beta signalling to regulate CCAAT/enhancer binding proteins and inhibit fat cell differentiation. J Cell Commun Signal 2015;9:27-36.

-39 Chen HX, Liu HY: The relationship between PPAR alpha gene polymorphism and type 2 diabetes mellitus. Medical review 2008;14:897-899.

40 Li Y, Jin DX, Xie WX, Wen LF, Chen WJ, Ding JY, Ren DC: PPAR-gamma and Wnt regulate the differentiation of MSCs into adipocytes and osteoblasts respectively. Curr Stem Cell Res Ther 2018;13:185-192. 


\section{Cellular Physiology Cell Physiol Biochem 2018;47:2458-2470 \begin{tabular}{l|l} 
DOI: 10.1159/000491619 & and Biochemistry \\
Published online: July 10, 2018 & $\begin{array}{l}\text { O 2018 The Author(s). Published by S. Karger AG, Basel } \\
\text { www.karger.com/cpb }\end{array}$
\end{tabular} \\ Li et al.: Identification and Characterization of CircRNAs in Pigs}

-41 Zhao Q, Lu YZ, Gan XQ Yu HY: Low magnitude high frequency vibration promotes adipogenic differentiation of bone marrow stem cells via P38 MAPK signal. Plos One 2017;12:e0172954.

-42 Prusty D, Park BH, Davis KE, Farmer SR: Activation of MEK/ERK signaling promotes adipogenesis by enhancing peroxisome proliferator-activated receptor gamma (PPARgamma) and C/EBPalpha gene expression during the differentiation of 3T3-L1 preadipocytes. J Biol Chem 2002;277:46226-46232.

-43 Reginato MJ, Krakow SL, Bailey ST, Lazar MA: Prostaglandins promote and block adipogenesis through opposing effects on peroxisome proliferator-activated receptor gamma. J Biol Chem 1998;273:1855-1858.

44 Xing Y, Yan F, Liu Y, Liu Y, Zhao Y: Matrine inhibits 3T3-L1 preadipocyte differentiation associated with suppression of ERK1/2 phosphorylation. Biochem Biophys Res Commun 2010;396:691-695.

45 Rondini EA, Mladenovic-Lucas L, Roush WR, Halvorsen GT, Green AE, Granneman JG: Novel Pharmacological Probes Reveal ABHD5 as a Locus of Lipolysis Control in White and Brown Adipocytes. J Pharmacol Exp Ther 2017;363:367-376.

-46 Flowers MT, Ntambi JM: Role of stearoyl-coenzyme A desaturase in regulating lipid metabolism. Curr Opin Lipidol 2008;19:248-256.

47 Miyazaki M, Kim YC, Ntambi JM: A lipogenic diet in mice with a disruption of the stearoyl-CoA desaturase 1 gene reveals a stringent requirement of endogenous monounsaturated fatty acids for triglyceride synthesis. J Lipid Res 2001;42:1018-1024.

48 Heldin CH, Miyazono K, Dijke PT: TGF-beta signalling from cell membrane to nucleus through SMAD proteins. Nature 1997;390:465-471.

49 Attisano L, Wrana JL: Signal transduction by the TGF-beta superfamily. Science 2002;296:1646-1647.

50 Hayashi H, Abdollah S, Qiu Y, Cai J, Xu YY, Grinnell BW, Richardson MA, Topper JN, Jr. Gimbrone MA, Wrana JL, Falb D: The MAD-related protein Smad7 associates with the TGFbeta receptor and functions as an antagonist of TGFbeta signaling. Cell 1997;89:1165-1173.

51 Choy L, Skillington J , Derynck R: Roles of autocrine TGF-beta receptor and Smad signaling in adipocyte differentiation. J Cell Biol 2000;149:667-682. 